This item was submitted to Loughborough's Research Repository by the author.

Items in Figshare are protected by copyright, with all rights reserved, unless otherwise indicated.

\title{
Kinetic equation for nonlinear wave-particle interaction: solution properties and asymptotic dynamics
}

PLEASE CITE THE PUBLISHED VERSION

https://doi.org/10.1016/j.physd.2018.12.007

PUBLISHER

(C) Elsevier

VERSION

AM (Accepted Manuscript)

\section{PUBLISHER STATEMENT}

This paper was accepted for publication in the journal Physica D: Nonlinear Phenomena and the definitive published version is available at https://doi.org/10.1016/j.physd.2018.12.007.

LICENCE

CC BY-NC-ND 4.0

\section{REPOSITORY RECORD}

Artemyev, A.V., Anatoly Neishtadt, and Alexei Vasiliev. 2019. "Kinetic Equation for Nonlinear Wave-particle Interaction: Solution Properties and Asymptotic Dynamics”. figshare. https://hdl.handle.net/2134/36523. 


\title{
Kinetic equation for nonlinear wave-particle interaction: solution properties and asymptotic dynamics
}

\author{
Anton Artemyev ${ }^{\mathrm{e}, \mathrm{d}}$, Anatoly Neishtadt ${ }^{\mathrm{f}, \mathrm{d}}$, Alexei Vasiliev ${ }^{\mathrm{d}}$ \\ ${ }^{a}$ Space Research Institute, Profsoyuznaya 84/32, Moscow 117997, Russia \\ ${ }^{b}$ Department of Earth, Planetary, and Space Sciences and Institute of Geophysics and \\ Planetary Physics, University of California, Los Angeles, California, USA \\ ${ }^{c}$ Department of Mathematical Sciences, Loughborough University, Loughborough LE11 \\ 3TU, UK
}

\begin{abstract}
We consider a kinetic equation describing evolution of the particle distribution function in a system with nonlinear wave-particle interactions (trappings into resonance and nonlinear scatterings). We study properties of its solutions and show that the only stationary solution is a constant, and that all solutions with smooth initial conditions tend to a constant as time grows. The resulting flattening of the distribution function in the domain of nonlinear interactions is similar to one described by the quasi-linear plasma theory, but the distribution evolves much faster. The results are confirmed numerically for a model problem.
\end{abstract}

Keywords: wave-particle nonlinear interaction, kinetic equation, resonances, distribution function

\footnotetext{
${ }^{*}$ Corresponding author. E-mail: valex@iki.rssi.ru
} 


\title{
Kinetic equation for nonlinear wave-particle interaction: solution properties and asymptotic dynamics
}

\author{
Anton Artemyev ${ }^{\mathrm{e}, \mathrm{d}}$, Anatoly Neishtadt ${ }^{\mathrm{f}, \mathrm{d}}$, Alexei Vasiliev ${ }^{\mathrm{d}}$ \\ ${ }^{d}$ Space Research Institute, Profsoyuznaya 84/32, Moscow 117997, Russia \\ 5 \\ ${ }^{e}$ Department of Earth, Planetary, and Space Sciences and Institute of Geophysics and \\ Planetary Physics, University of California, Los Angeles, California, USA \\ ${ }^{f}$ Department of Mathematical Sciences, Loughborough University, Loughborough LE11 \\ 3TU, UK
}

\section{Introduction}

10 change between particles and the energy transformation (e.g., from kinetic to thermal energy) are controlled by particle collisions, which lead to their momentum exchange [e.g., 1]. Rarefied space plasma systems are, however, collisionless, and hence other kinetic processes control the energy exchange ulation of particles can travel in space and interact with another, possibly quite distant, population. This interaction effectively connects particles which never physically collide with each other, and results in collisionless momentum exchanges. Therefore, understanding of the thermalization prointeraction [e.g., 2, 3].

The wave-particle resonant interaction plays a crucial role in the structure and dynamics of various space and laboratory plasma systems. In the nearEarth space environment, this interaction determines structure and dynamics of the collisionless bow shock [e.g., 4, and references therein], controls solar wind transport across the Earth magnetosphere boundary (magnetopause) [e.g., [5, and references therein], participates in magnetic energy release in the magnetotail reconnection [e.g., [6, and references therein], contributes to fieldaligned current dissipation [e.g., 7, and references therein], determines the

\footnotetext{
${ }^{*}$ Corresponding author. E-mail: valex@iki.rssi.ru
} 
radiation belt formation and dynamics [e.g., 8, and references therein], controls charged particle precipitation to aurora [e.g., 9, and references therein] and their acceleration in the aurora region [e.g., 10, and references therein].

The basic concept describing wave-particle interaction is the quasi-linear theory proposed in early 60s [11, 12]. It is based on the assumption of charged spectrum. However, as high-resolution wave measurements become available [e.g., 13, 14, 15, 16, 17, 18, 19, 20], the applicability of the quasi-linear theory becomes questionable. Intense coherent waves at the bow shock and in the Earth's magnetosphere can lead to effective momentum exchanges between dissipation. However, their resonant interaction with charged particles is nonlinear and cannot be described by the quasi-linear theory. This problem stimulates the development of new models and theories of the wave-particle resonant interactions and their contribution to large-scale plasma system structure and dynamics [e.g., 21, 22, 23, 24, 25, 26].

One of the perspective models of the nonlinear wave-particle interaction consists in generalization of the kinetic equation (generalized Fokker-Planck equation) in order to include effects of particle nonlinear scattering (nondiffusive drift in phase space [see, e.g., 27, 28, 29]) and nonlocal transport 50 (large jumps in the phase space due to phase trapping effect [see, e.g., 30, 31, 32 ). The combination of the Hamiltonian theory of perturbations in resonant systems [33] and the probabilistic approach for describing resonant systems results in such a kinetic (generalized Fokker-Planck) equation incorporating non-diffusive effects of the nonlinear wave-particle interaction. This equation successfully describes the evolution of the charged particle distribution in various space plasma systems and explains many important effects observed by spacecraft in the near-Earth plasma environment [see, e.g., discussion in $34,35,36$. In this paper we investigate general properties of this kinetic equation and its solutions. In Section 2 we demonstrate existence of a unique stationary solution of the kinetic equation, and show that it is a constant In Section 3 we consider a simplified version of the equation and construct its general solution; then we show that for any smooth initial distribution it tends to a constant solution as the time tends to infinity. In section 4 we use numerical simulations of the complete equation to test our analytical results derived in Sections 2 and 3. And finally in Section 5 we discuss the paper conclusions. 


\section{The kinetic equation and its properties}

Typically, resonant phenomena occur in systems with two scales of motion: fast and slow. Far from a resonance, one can average the dynamics

$$
\frac{\partial f}{\partial t}=-V(I) \frac{\partial f}{\partial I}-\frac{\partial V(I)}{\partial I}\left(f-f_{*}\right)+\frac{1}{2} \frac{\partial}{\partial I}\left(D(I) \frac{\partial f}{\partial I}\right), \text { if } I \geq I_{m} .
$$

Here smooth function $V(I)$ (the drift velocity due to scatterings) has the only extremum at $I=I_{m}$, is negative at $I_{-}<I<I_{+}$and zero otherwise. Function $D(I)$ (the diffusion coefficient due to scatterings) is also smooth 


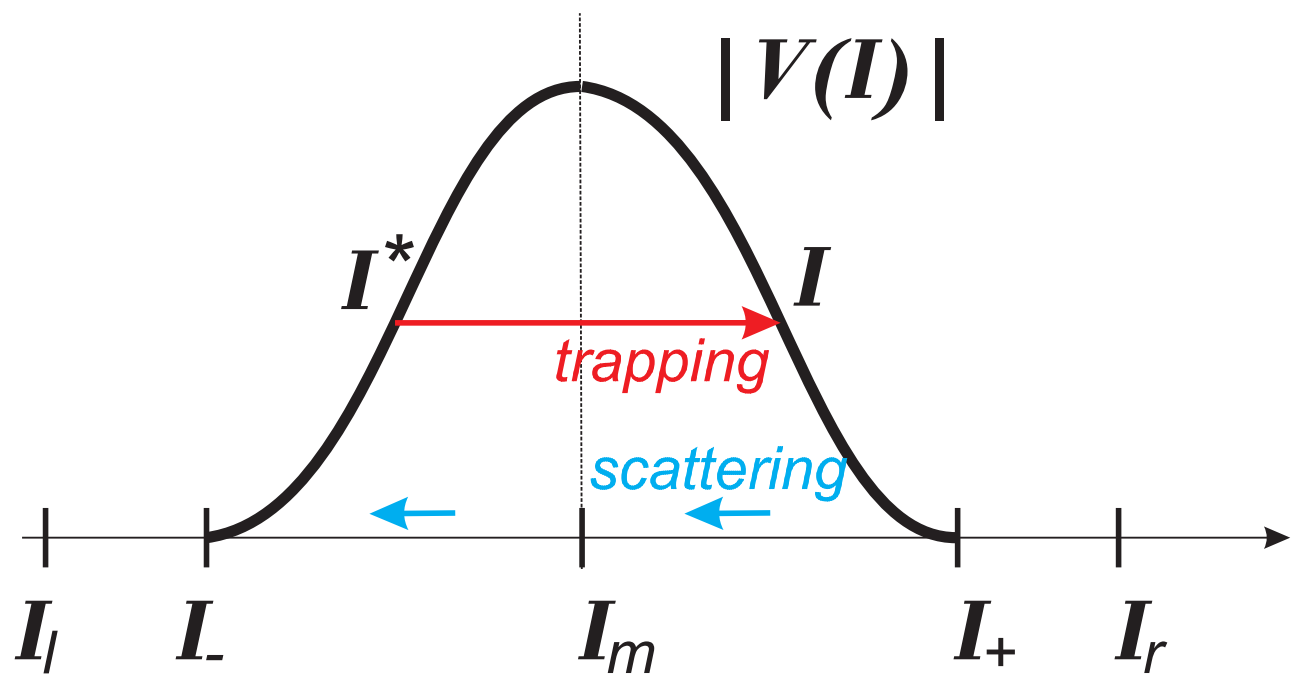

Figure 1: Schematic representation of the system dynamics. Fast variation (jump due to capture into the resonance) in $I$, shown with the red arrow, takes a particle from $I_{*} \in\left(I_{-}, I_{m}\right)$ to $I \in\left(I_{m}, I_{+}\right)$. Slow drift due to scatterings, shown with the blue arrows, transports particles in the opposite direction, from $I_{m}<I<I_{+}$to $I_{-}<I<I_{m}$. Note that $V(I) \leq 0$, hence $|V(I)|=-V(I)$.

and positive at $I_{l}<I<I_{r}$ and zero otherwise; $I_{l}<I_{-}<I_{m}<I_{+}<I_{r}$. Thus, for every value $V_{0}$ of function $V(I)$ (except for $V\left(I_{m}\right)$ ) there are two values of $I$ such that $V(I)=V_{0}$. For each $I>I_{m}$, we introduce $I_{*}<I_{m}$ such that $V\left(I_{*}\right)=V(I)$. Then $f_{*}$ in (2) denotes $f\left(t, I_{*}\right)$. Near $I=I_{+}$and $I=I_{-}$, function $V(I)$ has the following asymptotics: $V(I) \sim\left|I-I_{ \pm}\right|^{5 / 4}$ (see Appendix). We summarize the notations in Fig. 1.

\subsection{Conservation of the number of particles}

Equations (1)-(2) preserve the total number of particles:

$$
\begin{aligned}
\frac{\mathrm{d}}{\mathrm{d} t} \int_{I_{l}}^{I_{r}} f(t, I) \mathrm{d} I & =\int_{I_{l}}^{I_{r}} \frac{\partial f(t, I)}{\partial t} \mathrm{~d} I \\
& =\int_{I_{l}}^{I_{r}} \frac{1}{2} \frac{\partial}{\partial I}\left(D \frac{\partial f}{\partial t}\right) \mathrm{d} I+\int_{I_{-}}^{I_{m}}\left(-\frac{\partial}{\partial I}(V f)+f \frac{\partial V}{\partial I}\right) \mathrm{d} I \\
& +\int_{I_{m}}^{I_{+}}\left(-\frac{\partial}{\partial I}(V f)+f_{*} \frac{\partial V}{\partial I}\right) \mathrm{d} I \\
& =\int_{I_{-}}^{I_{m}} f(t, I) \frac{\partial V(I)}{\partial I} \mathrm{~d} I+\int_{I_{m}}^{I_{+}} f_{*}(t, \xi) \frac{\partial V(\xi)}{\partial \xi} \mathrm{d} \xi .
\end{aligned}
$$


Changing the integration variable in the second integral $\xi \mapsto I$ and using $V(I)=V(\xi), f(t, I)=f_{*}(t, \xi)$ we get

$$
\frac{\mathrm{d}}{\mathrm{d} t} \int_{I_{l}}^{I_{r}} f(t, I) \mathrm{d} I=\int_{I_{-}}^{I_{m}} f(t, I) \frac{\partial V(I)}{\partial I} \mathrm{~d} I+\int_{I_{m}}^{I_{-}} f(t, I) \frac{\partial V(I)}{\partial I} \mathrm{~d} I=0 .
$$

\subsection{A unique smooth stationary solution: $f=$ const}

Stationary solutions to (1)-(2) satisfy the following equations:

$$
\begin{array}{r}
-V(I) \frac{\partial f}{\partial I}+\frac{1}{2} \frac{\partial}{\partial I}\left(D(I) \frac{\partial f}{\partial I}\right)=0, \text { if } I \leq I_{m} ; \\
-V(I) \frac{\partial f}{\partial I}-\frac{\partial V(I)}{\partial I}\left(f-f_{*}\right)+\frac{1}{2} \frac{\partial}{\partial I}\left(D(I) \frac{\partial f}{\partial I}\right)=0, \text { if } I \geq I_{m} .
\end{array}
$$

For $I \leq I_{-}$and $I \geq I_{+}$we have $V(I)=0$. Hence, at these values of $I$, (4) is reduced to $\frac{\partial}{\partial I}\left(\bar{D}(I) \frac{\partial f}{\partial I}\right)=0$. Therefore $D(I) \frac{\partial f}{\partial I}=$ const. At $I=I_{l, r}$ function $D(I)$ is zero, hence the constant is zero and $\frac{\partial f}{\partial I}=0$. Thus, for $I \leq I_{-}$and $I \geq I_{+}$we find $f=$ const. We denote the value of $f$ at $I \leq I_{-}$ as $c_{-}$.

Consider now the range $I_{-} \leq I \leq I_{m}$. Denote $\frac{\partial f}{\partial I}=u$. We have:

$$
-V u+\frac{1}{2} \frac{\mathrm{d}}{\mathrm{d} I}(D u)=0 .
$$

Its solution is $u=\frac{c}{D} \exp \int(2 V / D) \mathrm{d} I$, where $c$ is a constant. We have shown above that $u\left(I_{-}\right)=\partial f /\left.\partial I\right|_{I_{-}}=0$. Therefore the constant $c=0$, and $\partial f / \partial I=0$ at $I \in\left[I_{-}, I_{m}\right]$. Hence, $f \equiv$ const $=c_{-}$.

Finally, consider the range $I_{m} \leq I \leq I_{+}$. For values of $I$ in this range, one should substitute $f_{*}=c_{-}$in $(4)$. Denote $\tilde{f}=f-c_{-}$. Then the second equation in (4) becomes

$$
-V \frac{\partial \tilde{f}}{\partial I}-\frac{\partial V}{\partial I} \tilde{f}+\frac{1}{2} \frac{\partial}{\partial I}\left(D \frac{\partial \tilde{f}}{\partial I}\right)=0
$$

And thus

$$
-V \tilde{f}+\frac{1}{2} D \frac{\partial \tilde{f}}{\partial I}=C
$$

where $C$ is a constant. At $I=I_{+}$we have $V\left(I_{+}\right)=0$ and $\partial \tilde{f} /\left.\partial I\right|_{I_{+}}=0$. Therefore, in (5), $C=0$, and we get

$$
-V \tilde{f}+\frac{1}{2} D \frac{\partial \tilde{f}}{\partial I}=0 .
$$


The last equation has the solution $\tilde{f}=c_{1} \exp \int(2 V / D) \mathrm{d} I$, where $c_{1}$ is a constant. By continuity of function $f$ we have $\tilde{f}\left(I_{m}\right)=0$. Hence $c_{1}=0$, $\tilde{f}=0$ and $f=c_{-}$.

It follows from the continuity of $f$ that $f=c_{-}$for $I \geq I_{+}$as well. Thus we have proven that the only smooth solution to (4) is $f=$ const.

\section{Stability of the constant stationary solution $(D=0)$}

To demonstrate that the constant stationary solution to (1)-(2) is stable, we restrict ourselves to the case of zero diffusion: $D(I) \equiv 0$. Doing so we keep in mind that in the systems with trappings into resonance and scatterings on resonance $D \ll V$ (in dimensionless units; see, e. g., [37, 38]). On the other hand, the diffusive term normally enhances stability. In the next section we numerically demonstrate the validity of the analytical results obtained for $D=0$ in the general case.

Consider equations

$$
\begin{gathered}
\frac{\partial f}{\partial t}=-V(I) \frac{\partial f}{\partial I}, \text { if } I \leq I_{m} ; \\
\frac{\partial f}{\partial t}=-V(I) \frac{\partial f}{\partial I}-\frac{\partial V(I)}{\partial I}\left(f-f_{*}\right), \text { if } I \geq I_{m} .
\end{gathered}
$$

Similarly to Section 2, equations (6)-(7) possess a unique smooth stationary solution $f=$ const. Below we prove that a smooth solution to (6)-(7) with initial condition $f(0, I)=f_{0}(I)$ tends to a constant as $t \rightarrow \infty$.

\subsection{General solution}

First, we construct the general solution to equations (6)-(7) on $I \in$ 140 $\left(I_{-}, I_{+}\right), t \in(0,+\infty)$.

Equations (6)-(7) are quasilinear PDEs. To find general solutions to these equations, we consider ODEs for the characteristic curves of (6)-(7) (see, e. g., [40]):

$$
\begin{aligned}
\frac{\mathrm{d} t}{1} & =\frac{\mathrm{d} I}{V(I)}=\frac{\mathrm{d} f}{0}, \text { if } I \leq I_{m} \\
\frac{\mathrm{d} t}{1} & =\frac{\mathrm{d} I}{V(I)}=\frac{\mathrm{d} f}{-\frac{\partial V}{\partial I}\left(f-f_{*}\right)}, \text { if } I \geq I_{m} .
\end{aligned}
$$


The first equality in (8) leads to

$$
t-\int_{I_{m}}^{I} \frac{\mathrm{d} \xi}{V(\xi)}=C_{1}
$$

where $C_{1}=$ const. Hence, the general solution to $(6)$ is

$$
f(t, I)=Q\left(t-\int_{I_{m}}^{I} \frac{\mathrm{d} \xi}{V(\xi)}\right), I \leq I_{m},
$$

145

where $Q(x)$ is an arbitrary smooth function.

From the first equality in (9) we get

$$
t-\int_{I_{m}}^{I} \frac{\mathrm{d} \xi}{V(\xi)}=C_{2}
$$

where $C_{2}=$ const. The second equality can be rewritten as a linear ODE

$$
\frac{\mathrm{d} f}{\mathrm{~d} I}=-\frac{1}{V} \frac{\partial V}{\partial I}\left(f-f_{*}\right), \quad I \geq I_{m} .
$$

The corresponding homogeneous equation

$$
\frac{\mathrm{d} f}{\mathrm{~d} I}=-\frac{1}{V} \frac{\partial V}{\partial I} f
$$

has a solution $f=C / V, C=$ const. To find the general solution to (11), we put $C=C(I)$ to find

$$
C(I)=\int_{I_{m}}^{I} \frac{\partial V(\xi)}{\partial \xi} f_{*}\left(C_{2}+\int_{I_{m}}^{\xi} \frac{\mathrm{d} \eta}{V(\eta)}, \xi\right) \mathrm{d} \xi .
$$

Substituting $C_{2}=t-\int_{I_{m}}^{I} \mathrm{~d} \eta / V(\eta)$ we find the general solution to 11 :

$$
\begin{aligned}
f(t, I) & =\frac{1}{V(I)} \int_{I_{m}}^{I} \frac{\partial V(\xi)}{\partial \xi} f_{*}\left(t+\int_{I}^{\xi} \frac{\mathrm{d} \eta}{V(\eta)}, \xi\right) \mathrm{d} \xi \\
& +\frac{1}{V(I)} P\left(t-\int_{I_{m}}^{I} \frac{\mathrm{d} \xi}{V(\xi)}\right), I \geq I_{m},
\end{aligned}
$$

where $P(x)$ is an arbitrary smooth function. 
A solution to (6)-(7) should be continuous at $I=I_{m}$. Thus, from (10) and $(12)$ we find

$$
P(t)=V_{m} Q(t)
$$

where $V_{m}=V\left(I_{m}\right)$.

Consider a value $I_{0}$ of $I$ such that $I_{0} \geq I_{m}$. Introduce function $\Lambda(I) \leq I_{m}$ such that $V\left(\Lambda\left(I_{0}\right)\right)=V\left(I_{0}\right)$. (In terms of trappings into resonance it means that a particle trapped at $I=\Lambda\left(I_{0}\right)$ escapes from resonance at $I=I_{0}$, see [37, 38.) Using this notation we have

$$
f_{*}(t, I)=f(t, \Lambda(I))=Q\left(t-\int_{I_{m}}^{\Lambda(I)} \frac{\mathrm{d} \xi}{V(\xi)}\right) .
$$

Now, from (12), (13), and (14) it is straightforward to obtain

$$
\begin{aligned}
f(t, I) & =\frac{1}{V(I)} \int_{I_{m}}^{I} \frac{\partial V(\xi)}{\partial \xi} Q\left(t-\int_{I_{m}}^{I} \frac{\mathrm{d} \eta}{V(\eta)}-\int_{\xi}^{\Lambda(\xi)} \frac{\mathrm{d} \eta}{V(\eta)}\right) \mathrm{d} \xi \\
& +\frac{V_{m}}{V(I)} Q\left(t-\int_{I_{m}}^{I} \frac{\mathrm{d} \xi}{V(\xi)}\right), I \geq I_{m} .
\end{aligned}
$$

\subsection{Solution with given initial conditions (Cauchy problem)}

Let $f_{0}(I)=f(0, I)$. From 110$)$ and $(15)$ at $t=0$ we have

$$
\begin{gathered}
f_{0}(I)=Q\left(-\int_{I_{m}}^{I} \frac{\mathrm{d} \xi}{V(\xi)}\right), I \leq I_{m}, \\
f_{0}(I)=\frac{1}{V(I)} \int_{I_{m}}^{I} \frac{\partial V(\xi)}{\partial \xi} Q\left(-\int_{I_{m}}^{I} \frac{\mathrm{d} \eta}{V(\eta)}-\int_{\xi}^{\Lambda(\xi)} \frac{\mathrm{d} \eta}{V(\eta)}\right) \mathrm{d} \xi \\
+\frac{V_{m}}{V(I)} Q\left(-\int_{I_{m}}^{I} \frac{\mathrm{d} \xi}{V(\xi)}\right), I \geq I_{m} .
\end{gathered}
$$

160 $(16),(17)$ provide a bounded and smooth solution to equations (6), (7). In
order to do this, we prove that there exists a unique smooth bounded function $Q$ satisfying (16), (17).

Note that $V(I) \leq 0$. Hence $\left(-\int_{I_{m}}^{I} \mathrm{~d} \xi / V(\xi)\right) \leq 0$ at $I \leq I_{m}$, and ${ }_{165}\left(-\int_{I_{m}}^{I} \mathrm{~d} \xi / V(\xi)\right) \geq 0$ at $I \geq I_{m}$. For values of $I$ close to $I_{ \pm}$we have 
$V(I) \sim\left|I-I_{ \pm}\right|^{5 / 4}$ (see Appendix) and hence the integral $\int_{I_{m}}^{I_{ \pm}} \mathrm{d} \xi / V(\xi)$ diverges. Therefore, given $f_{0}(I)$, equation (16) defines $Q(x)$ for all $x \in(-\infty, 0)$. Moreover, as $f_{0}(I)$ is bounded, (16) implies that $Q(x) \rightarrow f_{0}\left(I_{-}\right)$as $x \rightarrow-\infty$.

At $I \geq I_{m}$, function $Q(x)$ is defined by $(17)$. Introduce new integration 170 variable $x$ instead of $\xi$ :

$$
x=-\int_{I_{m}}^{I} \frac{\mathrm{d} \eta}{V(\eta)}-\int_{\xi}^{\Lambda(\xi)} \frac{\mathrm{d} \eta}{V(\eta)} .
$$

We have

$$
\mathrm{d} x=\left(-\frac{1}{V(\Lambda(\xi))} \frac{\partial \Lambda}{\partial \xi}+\frac{1}{V(\xi)}\right) \mathrm{d} \xi
$$

By its definition, function $\Lambda(\xi)$ satisfies $V(\xi)=V(\Lambda(\xi))$. Differentiating this equation, we obtain

$$
\frac{\partial \Lambda}{\partial \xi}=\frac{V^{\prime}(\xi)}{V^{\prime}(\Lambda(\xi))}
$$

where the prime denotes derivative of a function over its argument. Substituting into 19 , one obtains

$$
\frac{\partial V}{\partial \xi}=g(I, x) \mathrm{d} x
$$

where

$$
g(I, x)=\frac{1}{\left(V(\xi) V^{\prime}(\xi)\right)^{-1}-\left(V(\Lambda(\xi)) V^{\prime}(\Lambda(\xi))\right)^{-1}} .
$$

Equation (17) takes the form:

$$
V(I) f_{0}(I)=\int_{a(I)}^{b(I)} g(I, x) Q(x) \mathrm{d} x+V_{m} Q\left(-\int_{I_{m}}^{I} \frac{\mathrm{d} \xi}{V(\xi)}\right), \quad I \geq I_{m},
$$

where

$$
\begin{gathered}
a(I)=-\int_{I_{m}}^{I} \frac{\mathrm{d} \eta}{V(\eta)}>0, \\
b(I)=-\int_{I_{m}}^{\Lambda(I)} \frac{\mathrm{d} \eta}{V(\eta)}<0 .
\end{gathered}
$$

At $x \leq 0$ function $Q(x)$ is defined in terms of $f_{0}(I), I \leq I_{m}$ (see (16)). Therefore, the integral $\int_{0}^{b(I)} g(I, x) Q(x) \mathrm{d} x$ is a known function of $I$, which 
we denote as $h(I)$. Hence,

$$
-h(I)+V(I) f_{0}(I)=-\int_{0}^{a(I)} g(I, x) Q(x) \mathrm{d} x+V_{m} Q\left(-\int_{I_{m}}^{I} \frac{\mathrm{d} \xi}{V(\xi)}\right), \quad I \geq I_{m} .
$$

It follows from $(23,24)$ that $a(I) \rightarrow+\infty$ and $b(I) \rightarrow-\infty$ as $I \rightarrow I_{+}$. Using definitions of functions $g(I, x)$ and $h(I)$ and returning to the original integration variable $\xi$ one can see that $h(I)$ is bounded as $I \rightarrow I_{+}$. Moreover, the integral $\int_{0}^{a(I)} g(I, x) \mathrm{d} x$ converges as $I \rightarrow I_{+}$.

Equation 25) is a Volterra integral equation of the second kind with $a(I)$ considered as an independent variable (see, e.g., 40]). On any bounded segment $(0, A)$ of values of $a$ this equation has a unique continuous solution. Therefore, it has a unique continuous solution on any segment $\left(I_{m}, I_{k}\right)$ of values of $I$, where $I_{k}<I_{+}$.

We can rewrite (25) as

$$
\begin{aligned}
-h(I)+V(I) f_{0}(I) & =-\int_{0}^{A} g(I, x) Q(x) \mathrm{d} x-\int_{A}^{a(I)} g(I, x) Q(x) \mathrm{d} x \\
& +V_{m} Q\left(-\int_{I_{m}}^{I} \frac{\mathrm{d} \xi}{V(\xi)}\right), I \geq I_{m} .
\end{aligned}
$$

In the first integral in (26), $Q(x)$, is a known bounded function. Hence, this integral converges for any finite value of $A$ (i.e., for any $I<I_{+}$). Thus, we can rewrite the equation in the form

$$
-\tilde{h}(I)=-\frac{1}{V_{m}} \int_{A}^{a(I)} g(I, x) Q(x) \mathrm{d} x+Q\left(-\int_{I_{m}}^{I} \frac{\mathrm{d} \xi}{V(\xi)}\right),
$$

where $\tilde{h}(I)$ is a known function and we consider such values of $I$ that $a(I)>$ $A$. The integral $\int_{A}^{a(I)} g(I, x) \mathrm{d} x$ converges (see above) and is small at large enough values of $A$. Therefore, the operator

$$
Q(a(I))-\frac{1}{V_{m}} \int_{A}^{a(I)} g(I, x) Q(x) \mathrm{d} x
$$

is a contraction (see, e. g., [40]). Hence, equation (27) has a unique continuous bounded solution. Smoothness of $Q(x)$ at $x<0$ is due to smoothness of the initial condition $f_{0}(I)$ at $I<I_{m}$. Smoothness of $Q(x)$ at $x>0$ 
follows from its boundedness and equation 22 . Thus, the constructed solution $f(t, I)$ is smooth at $I<I_{m}$ and $I>I_{m}$. To prove smoothness of $f(t, I)$ at $I=I_{m}$ one can differentiate (10) and (15) with respect to $I$ and find the values of the both derivatives as $I \rightarrow I_{m}$. Taking into account that $\partial V / \partial I=0$ at $I=I_{m}$ it is straightforward to obtain that the both values of $\partial f / \partial I$ coincide.

\subsection{Behavior at $t \rightarrow \infty$}

Now we can study behavior of the constructed solution $f(t, I)$ as $t \rightarrow+\infty$.

According to the previous subsection, function $Q(x)$ is bounded. Therefore, there is a finite limit

$$
W=\lim _{I \rightarrow I_{+}} \int_{I_{m}}^{I} \frac{\partial V(\xi)}{\partial \xi} Q\left(-\int_{I_{m}}^{I} \frac{\mathrm{d} \eta}{V(\eta)}-\int_{\xi}^{\Lambda(\xi)} \frac{\mathrm{d} \eta}{V(\eta)}\right) \mathrm{d} \xi
$$

Indeed, $\partial V / \partial \xi=O\left(\left|\xi-I_{ \pm}\right|^{1 / 4}\right)$ if $\xi$ is close to $I_{ \pm}$, and $\partial V / \partial \xi=O\left(\left|\xi-I_{m}\right|\right)$ if $\xi$ is close to $I_{m}$. If the argument of $Q$ in (28) is negative, $Q$ is a known (in terms of $f_{0}(I)$, see $(16)$ ) bounded function. Taking into account that $V(I) \rightarrow 0$ as $I \rightarrow I_{+}$and $f_{0}(I)$ is bounded, we find from (17) and (28) that $Q(x) \rightarrow-W / V_{m}$ as $x \rightarrow+\infty$.

Consider now the $\operatorname{limit}_{t \rightarrow \infty} f(t, I)$ at a fixed $I \neq I_{ \pm}$. At $I \leq I_{m}$ we have

$$
f(t, I)=Q\left(t-\int_{I_{m}}^{I} \frac{\mathrm{d} \xi}{V(\xi)}\right) \rightarrow-\frac{W}{V_{m}}=\text { const, as } t \rightarrow \infty .
$$

Hence, for $I \geq I_{m}$ one has

$$
f_{*}(t, I) \rightarrow-\frac{W}{V_{m}}=\text { const, as } t \rightarrow \infty .
$$

Therefore, at $I \geq I_{m}$, we find from (12), (13) that

$$
f(t, I) \rightarrow \frac{1}{V(I)}\left(-\frac{W}{V_{m}} \int_{I_{m}}^{I} \frac{\partial V(\xi)}{\partial \xi} \mathrm{d} \xi-W\right)=-\frac{W}{V_{m}}, \text { as } t \rightarrow \infty .
$$

215

Thus, we have proved that $f(t, I) \rightarrow$ const as $t \rightarrow \infty$. Therefore, the constant solution to equations (6)-(7) is stable. In the following section we demonstrate that this is also valid for the kinetic equation (1)-(2) with $D(I) \neq 0$. 


\section{Numerical tests}

To verify our analytical results and confirm the main conclusion of stability of the stationary solution $f=$ const, we solve Eqs. (1) 2) numerically for a set of initial conditions, $f_{0}(I)$. Coefficients of Eqs. (1)-(2) and the specific form of $\Lambda(I)$ are defined from the analysis of Hamiltonian (32) (see Appendix). We use a sample function $V(I)=-\varepsilon^{1 / 2}\left(1-\left(I / I_{a}\right)^{2}\right)^{5 / 4}$ with $I_{ \pm}= \pm I_{a}$ and $I_{m}=0$ (thus, $\Lambda(I)=-I$ ) with $\varepsilon=10^{-3}$ (we put $V(I)=0$ for $\left.|I|>I_{a}\right)$. The diffusion coefficient is chosen in the form $D=D_{0} \varepsilon\left(1-I^{2}\right)$ and it is non-zero at $I \in[-1,1]$, i.e. if $I_{a}<1$ the $I$-range of nonlinear drift and trapping is shorter than the range of diffusion. The initial distribution is $f_{0}(I)=C_{0} \exp \left(-4\left(I-I_{0}\right)^{2}\right), C_{0}$ is defined by normalization $\int_{-1}^{+1} f_{0}(I) \mathrm{d} I=1$, and $I_{0}=0, \pm 1 / 2$ (this distribution is slightly modified around $I=I_{ \pm}$to satisfy the boundary conditions $\left.\mathrm{d} f /\left.\mathrm{d} I\right|_{I_{ \pm}}=0\right)$. This set of parameters assumes that particles (with $f(I, t)$ distribution) drift toward smaller $I$ and jump (due to trappings) from negative $I$ to positive $I$. Therefore, the initial distribution with $I_{0}=-1 / 2$ peaks within the region where charged particles can be trapped $(\mathrm{d} V / \mathrm{d} I<0)$. The evolution of this distribution allows both drift and jumps from the very beginning. The initial distribution with $I_{0}=1 / 2$ peaks within the region where trappings are not possible $(\mathrm{d} V / \mathrm{d} I>0)$. This distribution should first drift to the region of $I<0$ (where $\mathrm{d} V / \mathrm{d} I<0$ ), and only then the jumps due to trappings can start. The initial distribution with $I_{0}=0$ peaks at $\mathrm{d} V / \mathrm{d} I=0$. The evolution of this distribution should resemble spreading, because the distribution drifts to smaller $I$ (larger $|I|$ ) and at the same time some portion of these particles (with $I<0$ ) is transported towards large $I$.

We consider two sets of solutions: with $I_{a}=1$ (Fig. 2) and with $I_{a}=1 / \sqrt{2}$ (Fig. 3). In the first set of solutions, the distribution $f(t, I)$ changes slowly near the boundary $I_{ \pm}$because both $V$ and $D$ vanish there, but eventually this evolution form the flat distribution $f \sim$ const. In the second set of solutions, the boundary of the $I$-range of nonlinear drift and trappings is located at $I= \pm I_{a}$ where the diffusion is still quite strong (not vanishing). This results in the absence of peculiarities (local maxima) of particle distribution at this boundary: the nonlinear processes (drift and trappings) rapidly form $f \sim$ const distribution at $|I|<I_{a}$ and then slow diffusion moves boundaries of this distribution toward the system boundary, $I= \pm 1$.

Figures 2, 3 show results of numerical solutions of Eqs. (1)-( 2). Left 

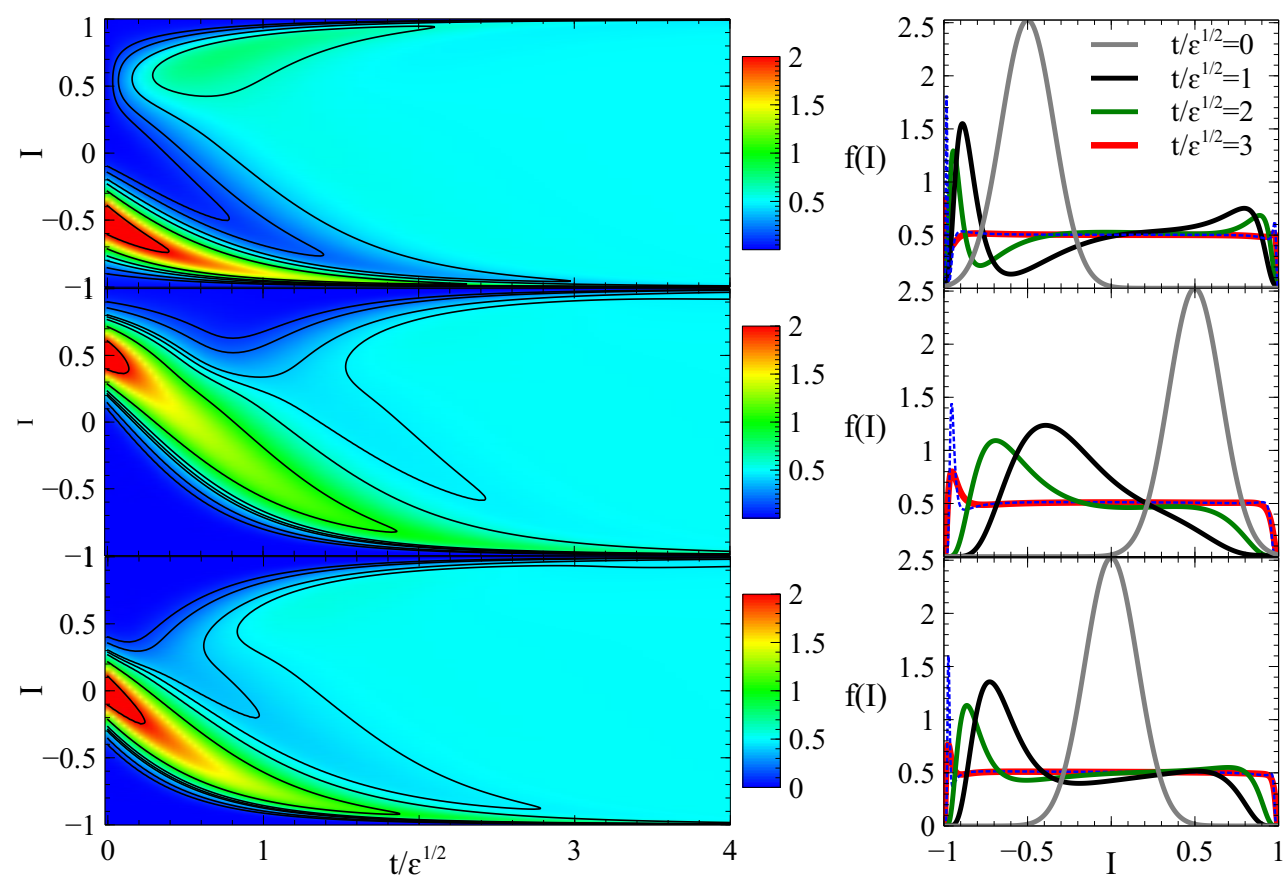

Figure 2: Solutions of Eqs. (1)-(2) with three initial distributions $f_{0}(I)=C_{0} \exp (-4(I-$ $\left.I_{0}\right)^{2}$ ) with $I_{0}=-1 / 2$ (top panels), with $I_{0}=1 / 2$ (middle panels), and with $I_{0}=0$ (bottom panels). Left panels show 2D-distributions $f(t, I)$ and right panels show $f(t, I)$ at four time moments. Blue dashed lines show $f(t, I)$ at $t / \sqrt{\varepsilon}=3$ for the solution with $D_{0}=1 / 10$ (all other curves and $2 \mathrm{D}$-distributions are for solutions with $D_{0}=1$ ). The boundary conditions are $\mathrm{d} f / \mathrm{d} I=0$ at $I= \pm 1$, and the drift velocity is $V(I)=-\varepsilon^{1 / 2}\left(1-\left(I / I_{a}\right)^{2}\right)^{5 / 4}$ with $I_{a}=1$, i.e. the diffusion and nonlinear drift (and trapping) are acting on the same $I$ range.

panels demonstrate 2D-distributions $f(t, I)$, and right panels show $f(t, I)$ at four time moments. The initial distribution determines the beginning of the evolution, but after $t / \sqrt{\varepsilon} \sim 2$ distribution $f(t, I)$ becomes quite flat in all three examples. The diffusion contributes to $f$ spreading, but does not change the general evolution which is determined by drift and trapping effects (compare red and blue curves at the right panels; these curves are plotted for solutions with $D_{0}=1$ and $\left.D_{0}=1 / 10\right)$. Thus, the numerical solution confirms our main conclusion: independently on initial distribution $f_{0}(I)$ the solution of Eqs. (1)-(2) tends to the uniform one $f=$ const. 

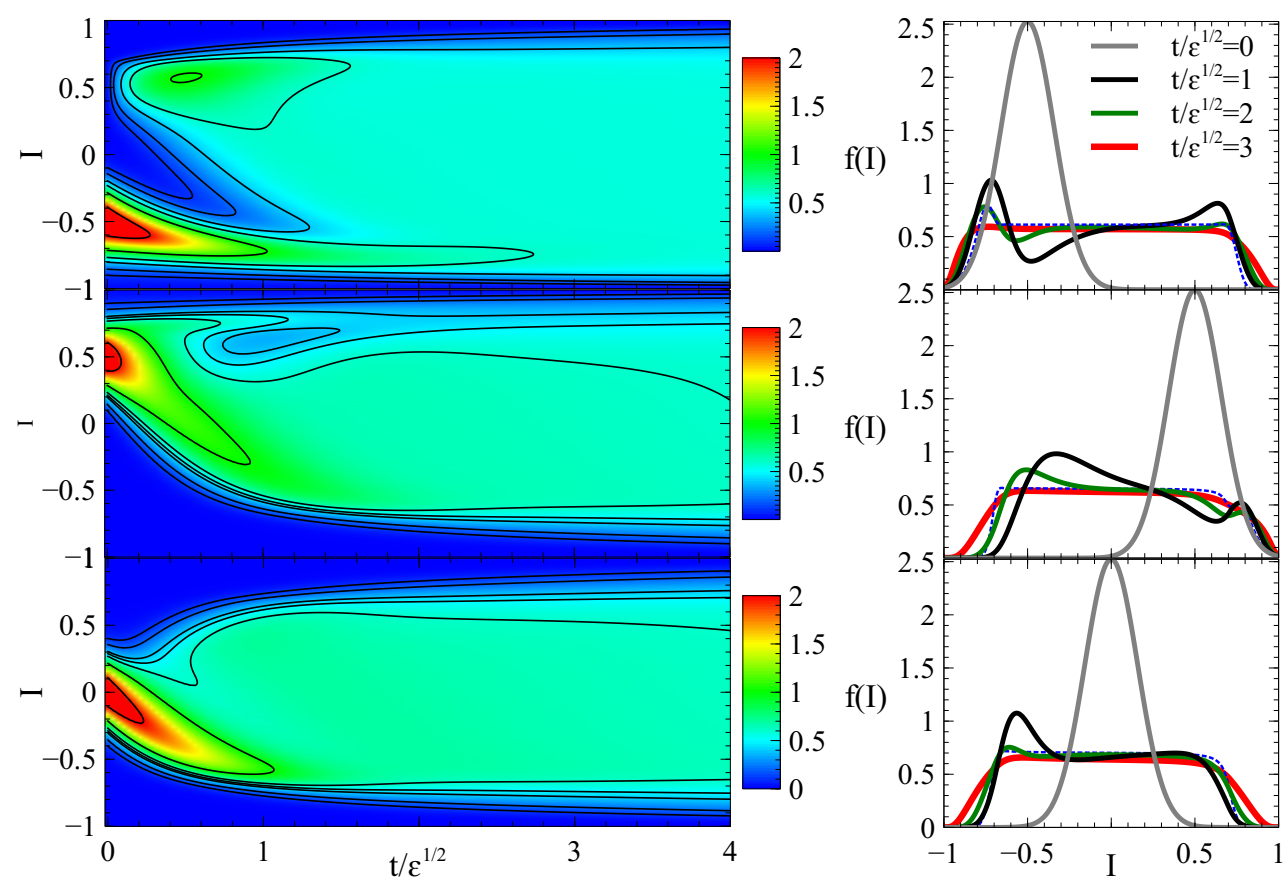

Figure 3: Solutions of Eqs. (1)-(2) with three initial distributions $f_{0}(I)=C_{0} \exp (-4(I-$ $\left.I_{0}\right)^{2}$ ) with $I_{0}=-1 / 2$ (top panels), with $I_{0}=1 / 2$ (middle panels), and with $I_{0}=0$ (bottom panels). Left panels show 2D-distributions $f(t, I)$ and right panels show $f(t, I)$ at four time moments. Blue dashed lines show $f(t, I)$ at $t / \sqrt{\varepsilon}=3$ for the solution with $D_{0}=1 / 10$ (all other curves and 2D-distributions are for solutions with $D_{0}=1$ ). The boundary conditions are $\mathrm{d} f / \mathrm{d} I=0$ at $I= \pm 1$, and the drift velocity is $V(I)=-\varepsilon^{1 / 2}\left(1-\left(I / I_{a}\right)^{2}\right)^{5 / 4}$ with $I_{a}=1 / \sqrt{2}$, i.e. the nonlinear drift and trappings act on a shorter $I$-range than the diffusion. 


\section{Discussion and conclusions}

265

In this paper we show that the kinetic equation which includes nonlinear effects of particle trapping and scattering by high-amplitude waves has a unique stationary solution, $f=$ const, and all solutions with smooth initial conditions tend to constant as $t \rightarrow \infty$. Thus, despite significant difference between this equation (i.e., Eqs. (1)-(2) ) and the classical diffusion equation describing the quasi-linear wave-particle interaction [e.g., 11, 12, the solutions for both equations tend to a constant. The main difference between the nonlinear resonant interaction including trappings and drift and the quasilinear diffusion is the time-scale required to form a constant solution. The typical time-scale of the nonlinear processes is $\sim 1 / V \sim \varepsilon^{-1 / 2}$, whereas the time-scale of diffusion varies from $1 / D \sim 1 / \varepsilon$ [e.g., 41] to $1 / D \sim 1 / \varepsilon^{2}$ [42] depending on the characteristics of a particular plasma system. Thus, in the $I$-range where nonlinear wave-particle interaction is possible, the solution $f=$ const is formed much faster than in the $I$-range where only diffusion affects the particle distribution (see, e.g., Fig. 3). In realistic plasma systems where a value $(I+$ const $)$ plays the role of the particle energy [see, e.g., 43, 44, 38], distributions $f(t, I)$ have a typical shape of energy spectra, i.e. $f$ decreases as $I$ increase. For such distributions, the nonlinear wave-particle interaction within a limited $I$-range results in formation of a plateau and the following evolution of this plateau is due to the diffusion (see, for example, Fig. (4). Such distributions with a energy-limited plateau are usually observed in the near-Earth plasma systems where the nonlinear wave-particle interaction is supposed to be strong [e.g., 45, 46, 47]. Note also that such regions of flattened distribution might be of interest in problems of tailoring barriers in the phase space in laboratory plasmas (c.f., e.g., [48]). One should also mention that on time scales of order less than $\varepsilon^{-1 / 2}$, i. e. in the process of relaxation, the nonlinear effects can produce localized structures depending on the specific properties of the system. An example of such structures in a model problem can be found in [49].

In conclusion, we have shown that the kinetic equation including effects of the nonlinear wave-particle resonant interaction (scattering-induced drift and trappings) has a unique stationary solution $f=$ const. This solution is stable, and any solution $f(t, I)$ with smooth initial conditions tends to constant as $t \rightarrow \infty$. We used numerical simulations to illustrate that particle diffusion does not significantly affect these conclusions. Obtained results could be useful for analysis of various plasma systems where the nonlinear 


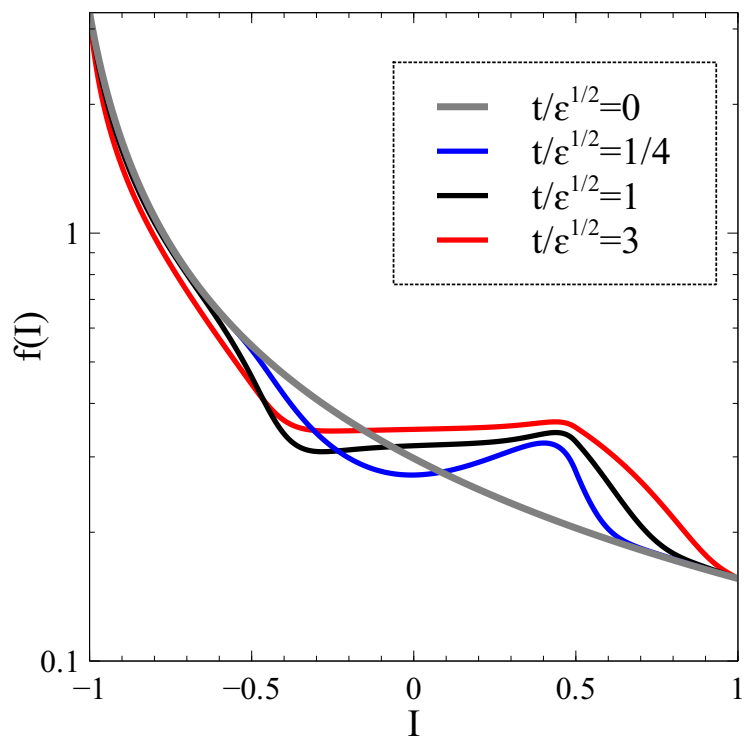

Figure 4: Solutions of Eqs. (1)-(2) for the initial distribution $f_{0}(I)=C_{0} /\left(I_{0}+I\right)$ with $I_{0}=11 / 10$. The boundary conditions are $d f / d I=0$ at $I= \pm 1$, and the drift velocity equation is $V(I)=-\varepsilon^{1 / 2}\left(1-\left(I / I_{a}\right)^{2}\right)^{5 / 4}$ with $I_{a}=1 / 2$, i.e. the nonlinear drift and trappings act on a shorter $I$-range than the diffusion. 
wave-particle interaction plays an important role.

\section{Appendix}

Function $V(I)$ is proportional to the area bounded by the separatrix loop on the phase portrait of the system near the resonance (see [37, 38, 39]). The corresponding Hamiltonian (called "pendulum-like" Hamiltonian, see, e. g., [50, 51]) has the form

$$
F=\frac{1}{2} g(I) K^{2}-A(I) \sin \varphi+\beta(I) \varphi
$$

where $K, \varphi$ are canonically conjugate variables and $I$ can be considered as a parameter; $g, A, \beta$ are functions of $I$; one may assume that they are positive. (Note that in 39] the variable corresponding to our $I$ is denoted as $J$ ). If $A(I)>\beta(I)$, there is the separatrix on the phase portrait. At $I=I_{ \pm}$one has $A=\beta$, and the saddle-center bifurcation occurs. Consider for definiteness $I=I_{+}$. At $I=I_{+}$, the separatrix loop disappears at $\varphi=0$ (see Fig 5). Expanding Hamiltonian $F$ near $I=I_{+}, \varphi=0$ one obtains in the main approximation

$$
F=\frac{1}{2} g\left(I_{+}\right) K^{2}-\delta \varphi+\tilde{A} \varphi^{3}
$$

315 where $\delta=\left(A^{\prime}\left(I_{+}\right)-\beta^{\prime}\left(I_{+}\right)\right)\left(I-I_{+}\right) ; \tilde{A}=A\left(I_{+}\right) / 6$. The area inside the separatrix loop on the phase portrait $($ at $\delta>0)$ is

$$
S(\delta)=2 \int_{\varphi_{1}}^{\varphi_{2}} K \mathrm{~d} \varphi=2 \int_{\varphi_{1}}^{\varphi_{2}} \sqrt{\frac{2}{g}\left(F_{s}+\delta \varphi-\tilde{A} \varphi^{3}\right)} \mathrm{d} \varphi
$$

where $\varphi_{1}, \varphi_{2} \sim \sqrt{\delta}$ are the zeros of the integrand, and $F_{s} \sim \delta^{3 / 2}$ is the value of the Hamiltonian $F$ at the saddle point. At $\delta \ll 1$, one obtains $S(\delta) \sim \delta^{5 / 4}$. Therefore, $V(I)$ at $I$ close to $I_{ \pm}$has the asymptotics $V \sim\left|I-I_{ \pm}\right|^{5 / 4}$. 

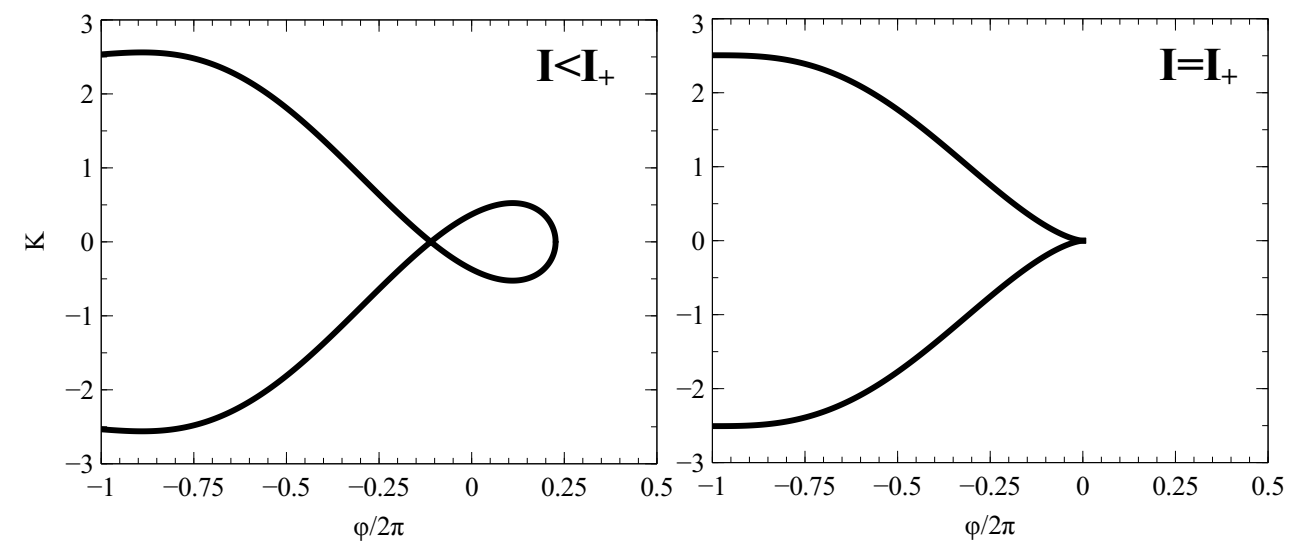

Figure 5: Phase portraits of system (32) for $I<I_{+}$(left panel) and $I=I_{+}$(right panel).

[6] M. Fujimoto, I. Shinohara, H. Kojima, Reconnection and Waves: A Review with a Perspective, Space Sci. Rev. 160 (2011) 123-143. doi: 10.1007/s11214-011-9807-7. 
340

[7] R. L. Lysak, Electrodynamic coupling of the magnetosphere and ionosphere, Space Sci. Rev. 52 (1990) 33-87. doi:10.1007/BF00704239

[8] R. M. Thorne, Radiation belt dynamics: The importance of waveparticle interactions, Geophys Res. Lett. 372 (2010) 22107. doi: 10.1029/2010GL044990.

[9] B. Ni, R. M. Thorne, X. Zhang, J. Bortnik, Z. Pu, L. Xie, Z.-j. $\mathrm{Hu}$, D. Han, R. Shi, C. Zhou, X. Gu, Origins of the Earth's Diffuse Auroral Precipitation, Space Sci. Rev. 200 (2016) 205-259. doi: 10.1007/s11214-016-0234-7.

[10] C. E. J. Watt, R. Rankin, Alfvén Wave Acceleration of Auroral Electrons in Warm Magnetospheric Plasma, Washington DC American Geophysical Union Geophysical Monograph Series 197 (2012) 251-260. doi:10.1029/2011GM001171.

[11] W. E. Drummond, D. Pines, Nonlinear stability of plasma oscillations, Nuclear Fusion Suppl. 3 (1962) 1049-1058.

[12] A. A. Vedenov, E. Velikhov, R. Sagdeev, Quasilinear theory of plasma oscillations, Nuclear Fusion Suppl. 2 (1962) 465-475.

[13] H. Matsumoto, H. Kojima, T. Miyatake, Y. Omura, M. Okada, I. Nagano, M. Tsutsui, Electrotastic Solitary Waves (ESW) in the magnetotail: BEN wave forms observed by GEOTAIL, Geophys Res. Lett. 21 (1994) 2915-2918. doi:10.1029/94GL01284.

[14] E. V. Panov, J. Büchner, M. Fränz, A. Korth, S. P. Savin, K.-H. Fornaçon, I. Dandouras, H. Rème, CLUSTER observation of collisionless transport at the magnetopause, Geophys Res. Lett. 33 (2006) 15109. doi:10.1029/2006GL026556.

[15] S. D. Bale, F. S. Mozer, Measurement of Large Parallel and Perpendicular Electric Fields on Electron Spatial Scales in the Terrestrial Bow Shock, Physical Review Letters 98 (20) (2007) 205001. doi: 10.1103/PhysRevLett.98.205001.

[16] C. Cattell, J. R. Wygant, K. Goetz, K. Kersten, P. J. Kellogg, T. von Rosenvinge, S. D. Bale, I. Roth, M. Temerin, M. K. Hudson, R. A. Mewaldt, M. Wiedenbeck, M. Maksimovic, R. Ergun, M. Acuna, C. T. 
Russell, Discovery of very large amplitude whistler-mode waves in Earth's radiation belts, Geophys Res. Lett. 35 (2008) 1105. doi: 10.1029/2007GL032009.

[17] C. M. Cully, J. W. Bonnell, R. E. Ergun, THEMIS observations of longlived regions of large-amplitude whistler waves in the inner magnetosphere, Geophys Res. Lett. 35 (2008) 17. doi:10.1029/2008GL033643.

[18] Y. V. Khotyaintsev, A. Vaivads, M. André, M. Fujimoto, A. Retinò, C. J. Owen, Observations of Slow Electron Holes at a Magnetic Reconnection Site, Physical Review Letters 105 (16) (2010) 165002. doi:10.1103/ PhysRevLett.105.165002.

[19] C. C. Chaston, Y. Yao, N. Lin, C. Salem, G. Ueno, Ion heating by broadband electromagnetic waves in the magnetosheath and across the magnetopause, J. Geophys. Res. 118 (2013) 5579-5591. doi:10.1002/ jgra.50506.

[20] F. D. Wilder, R. E. Ergun, K. A. Goodrich, M. V. Goldman, D. L. Newman, D. M. Malaspina, A. N. Jaynes, S. J. Schwartz, K. J. Trattner, J. L. Burch, M. R. Argall, R. B. Torbert, P.-A. Lindqvist, G. Marklund, O. Le Contel, L. Mirioni, Y. V. Khotyaintsev, R. J. Strangeway, C. T. Russell, C. J. Pollock, B. L. Giles, F. Plaschke, W. Magnes, S. Eriksson, J. E. Stawarz, A. P. Sturner, J. C. Holmes, Observations of whistler mode waves with nonlinear parallel electric fields near the dayside magnetic reconnection separatrix by the Magnetospheric Multiscale mission, Geophys Res. Lett. 43 (2016) 5909-5917. doi:10.1002/2016GL069473.

[21] Y. Omura, Y. Miyashita, M. Yoshikawa, D. Summers, M. Hikishima, Y. Ebihara, Y. Kubota, Formation process of relativistic electron flux through interaction with chorus emissions in the Earth's inner magnetosphere, J. Geophys. Res. 120 (2015) 9545-9562. doi:10.1002/ 2015JA021563.

[22] A. Osmane, L. B. Wilson, III, L. Blum, T. I. Pulkkinen, On the Connection between Microbursts and Nonlinear Electronic Structures in Planetary Radiation Belts, Astrophys. J. 816 (2016) 51. doi: 10.3847/0004-637X/816/2/51. 
[23] Y.-K. Hsieh, Y. Omura, Nonlinear dynamics of electrons interacting with oblique whistler mode chorus in the magnetosphere, J. Geophys. Res. 122 (2017) 675-694. doi:10.1002/2016JA023255.

[24] D. R. Shklyar, Energy transfer from lower energy to higher-energy electrons mediated by whistler waves in the radiation belts, J. Geophys. Res. 122 (1) (2017) 640-655. doi:10.1002/2016JA023263.

[25] A. G. Demekhov, U. Taubenschuss, O. Santolík, Simulation of VLF chorus emissions in the magnetosphere and comparison with THEMIS spacecraft data, J. Geophys. Res. 122 (2017) 166-184. doi:10.1002/ 2016JA023057.

[26] A. V. Artemyev, A. I. Neishtadt, D. L. Vainchtein, A. A. Vasiliev, I. Y. Vasko, L. M. Zelenyi, Trapping (capture) into resonance and scattering on resonance: Summary of results for space plasma systems, Communications in Nonlinear Science and Numerical Simulations 65 (2018) 111-160. doi:10.1016/j.cnsns.2018.05.004.

[27] D. Shklyar, H. Matsumoto, Oblique Whistler-Mode Waves in the Inhomogeneous Magnetospheric Plasma: Resonant Interactions with Energetic Charged Particles, Surveys in Geophysics 30 (2009) 55-104. doi:10.1007/s10712-009-9061-7.

[28] A. V. Artemyev, A. A. Vasiliev, D. Mourenas, O. V. Agapitov, V. V. Krasnoselskikh, Electron scattering and nonlinear trapping by oblique whistler waves: The critical wave intensity for nonlinear effects, Physics of Plasmas 21 (10) (2014) 102903. doi:10.1063/1.4897945.

[29] J. M. Albert, X. Tao, J. Bortnik, Aspects of Nonlinear Wave-Particle Interactions, in: D. Summers, I. U. Mann, D. N. Baker, M. Schulz (Eds.), Dynamics of the Earth's Radiation Belts and Inner Magnetosphere, American Geophysical Union, 2013. doi:10.1029/2012GM001324.

[30] Y. Omura, N. Furuya, D. Summers, Relativistic turning acceleration of resonant electrons by coherent whistler mode waves in a dipole magnetic field, J. Geophys. Res. 112 (2007) 6236. doi:10.1029/2006JA012243.

[31] A. G. Demekhov, V. Y. Trakhtengerts, M. Rycroft, D. Nunn, Efficiency of electron acceleration in the Earth's magnetosphere by whistler mode 
waves, Geomagnetism and Aeronomy 49 (2009) 24-29. doi:10.1134/ S0016793209010034.

[32] A. Artemyev, O. Agapitov, D. Mourenas, V. Krasnoselskikh, V. Shastun, F. Mozer, Oblique whistler-mode waves in the earth's inner magnetosphere: Energy distribution, origins, and role in radiation belt dynamics, Space Science Reviews 200 (2016) 261-355. doi:10.1007/ s11214-016-0252-5.

[33] V. I. Arnold, V. V. Kozlov, A. I. Neishtadt, Mathematical Aspects of Classical and Celestial Mechanics, 3rd Edition, Dynamical Systems III. Encyclopedia of Mathematical Sciences, Springer-Verlag, New York, 2006.

[34] A. V. Artemyev, A. I. Neishtadt, A. A. Vasiliev, D. Mourenas, Long-term evolution of electron distribution function due to nonlinear resonant interaction with whistler mode waves, Journal of Plasma Physics 84 (2018) 905840206. doi:10.1017/S0022377818000260.

[35] X.-J. Zhang, R. Thorne, A. Artemyev, D. Mourenas, V. Angelopoulos, J. Bortnik, C. A. Kletzing, W. S. Kurth, G. B. Hospodarsky, Properties of intense field-aligned lower-band chorus waves: Implications for nonlinear wave-particle interactions, J. Geophys. Res. 123 (7) 5379-5393. doi:10.1029/2018JA025390.

[36] D. Mourenas, X.-J. Zhang, A. V. Artemyev, V. Angelopoulos, R. M. Thorne, J. Bortnik, A. I. Neishtadt, A. A. Vasiliev, Electron Nonlinear Resonant Interaction With Short and Intense Parallel Chorus Wave Packets, J. Geophys. Res. 123 (2018) 4979-4999. doi:10.1029/ 2018JA025417.

[37] A. V. Artemyev, A. I. Neishtadt, A. A. Vasiliev, D. Mourenas, Kinetic equation for nonlinear resonant wave-particle interaction, Physics of Plasmas 23 (9) (2016) 090701. doi:10.1063/1.4962526.

[38] A. V. Artemyev, A. I. Neishtadt, A. A. Vasiliev, D. Mourenas, Probabilistic approach to nonlinear wave-particle resonant interaction, Phys. Rev. E95 (2) (2017) 023204. doi:10.1103/PhysRevE.95.023204. 
[39] A. V. Artemyev, A. I. Neishtadt, A. A. Vasiliev, D. Mourenas, Kinetic equation for systems with resonant captures and scatterings, ArXiv eprintsarXiv: 1710.04489 .

[40] R. Courant, D. Hilbert, Methods of Mathematical Physics, Interscience Publishers, New York and London, 1953.

[41] V. I. Karpman, D. R. Shkliar, Particle precipitation caused by a single whistler-mode wave injected into the magnetosphere, Planatary Space Science 25 (1977) 395-403. doi:10.1016/0032-0633(77)90055-1.

[42] C. F. Kennel, F. Engelmann, Velocity Space Diffusion from Weak Plasma Turbulence in a Magnetic Field, Physics of Fluids 9 (1966) 23772388. doi:10.1063/1.1761629.

[43] D. R. Shklyar, Stochastic motion of relativistic particles in the field of a monochromatic wave, Sov. Phys. JETP 53 (1981) 1197-1192.

[44] J. M. Albert, Cyclotron resonance in an inhomogeneous magnetic field, Physics of Fluids B 5 (1993) 2744-2750. doi:10.1063/1.860715.

[45] K. Min, K. Liu, W. Li, Signatures of electron Landau resonant interactions with chorus waves from THEMIS observations, J. Geophys. Res. 119 (2014) 5551-5560. doi:10.1002/2014JA019903.

[46] J. Li, J. Bortnik, X. An, W. Li, R. M. Thorne, M. Zhou, W. S. Kurth, G. B. Hospodarsky, H. O. Funsten, H. E. Spence, Chorus Wave Modulation of Langmuir Waves in the Radiation Belts, Geophys Res. Lett. 44 (2017) 11. doi:10.1002/2017GL075877.

[47] S. Kurita, Y. Miyoshi, S. Kasahara, S. Yokota, Y. Kasahara, S. Matsuda, A. Kumamoto, A. Matsuoka, I. Shinohara, Deformation of electron pitch angle distributions caused by upper-band chorus observed by the arase satellite, Geophys Res. Lett. 45 (2018) 7996-8004. doi: 10.1029/2018GL079104.

[48] S. Ogawa, X. Leoncini, A. Vasiliev, X. Garbet, Tailoring steep density profile with unstable points, Physics Letters A 383 (2019) 3539, //doi: 10.1016/j.physleta.2018.09.014. 
[49] X. Leoncini, A. Vasiliev, A. Artemyev, Resonance controlled transport in phase space, Physica D 364 (2018) 2226, //doi:10.1016/j.physd. 2017.09.010.

[50] A. I. Neishtadt, On Adiabatic Invariance in Two-Frequency Systems, in Hamiltonian Systems with Three or More Degrees of Freedom, ed. Simo C., NATO ASI Series C. Dordrecht: Kluwer Acad. Publ. 533 (1999) 193-213. doi:10.1063/1.166236.

[51] A. I. Neishtadt, A. A. Vasiliev, Destruction of adiabatic invariance at resonances in slow fast Hamiltonian systems, Nuclear Instruments and Methods in Physics Research A 561 (2006) 158-165. doi:10.1016/j. nima.2006.01.008. 